\title{
PERFIL DE USUÁRIOS DO SERVIÇO DE FISIOTERAPIA EM UMA UNIDADE BÁSICA DE SAÚDE
}

Mayane Santos Arantes, Paula de Barros Manfrim, Lucas Oliveira Klebis, Elaine Aparecida Lozano da Silva, Edna Maria do Carmo, Eliane Ferrari Chagas.

Universidade Estadual Paulista - UNESP, Programa de Pós-Graduação em Fisioterapia - Residência em Saúde, Presidente Prudente, SP. e-mail: may ane santos@hotmail.com

\section{RESUMO}

A atuação do Fisioterapeuta vem ganhando espaço na saúde pública por desenvolver assistência curativa e preventiva, trabalhando tanto na promoção de saúde como na reabilitação. Esta ação depende da demanda existente e conhecer a população assistida é importante para melhorar o processo de atendimento e organização dentro deste serviço. O objetivo foi avaliar o perfil de usuários atendidos no serviço de Fisioterapia em uma Unidade Básica de Saúde para conhecer a demanda local e as necessidades do serviço. Trata-se de estudo retrospectivo, realizado por análise de prontuários dos pacientes que procuraram o serviço de Fisioterapia. Houve 212 pessoas atendidas no setor de Fisioterapia, sendo 67,66\% mulheres e $32,34 \%$ homens. A média de idade foi de $48,8 \pm 18,1$ anos sendo trabalhadores do lar, faxineiros, motoristas, operadores de máquinas e construção civil. Houve maior prevalência de lesões ortopédicas e traumáticas e houve 114 casos crônicos e 78 de lesões agudas em todas as áreas.

Palavras-chave: Perfil de Saúde, Modalidade de Fisioterapia, Saúde Pública.

\section{PROFILE OF USERS IN PHYSICAL THERAPY SERVICE IN A BASIC HEALTH UNIT}

\begin{abstract}
The role of the physiotherapist has been improvement in public health for developing curative and preventive care, working in both health promotion and rehabilitation. This action depends upon the demand and it is important to know which is the population attended for improving the process of care and organization in this service. The objective was to evaluate the profile of patients attended in Physical Therapy services in a Basic Health Unit to understand local demand and the needs of the service. It was a retrospective study by review of medical records of patients seeking physical therapy services. There were 212 people attended in Physical Therapy service, $67.66 \%$ women and $32.34 \%$ men. The mean age was 48.8 \pm 18.1 years old. They were home workers, maids, drivers, machines and construction works. There was a higher prevalence of orthopedic and traumatic injuries and there were 114 chronic cases and 78 of acute injuries in all areas.
\end{abstract}

Keywords: Health Profile, Physical Therapy Modalities, Public Health. 


\section{INTRODUÇÃO}

O Sistema Único de Saúde (SUS) foi criado pela Constituição em 1988, apresentando como princípios e diretrizes o atendimento a saúde de forma universal, equânime e integral, sendo capaz de oferecer a população o atendimento público ${ }^{1}$. Dentro deste conceito, os atendimentos em Unidade Básica de Saúde (UBS) representam um dos serviços realizados pelo SUS em que de direciona, principalmente, o atendimento primário e básico em saúde. $O$ atendimento primário é caracterizado como prevenção e promoção à saúde, no entanto, diante do perfil e demanda da população, os serviços da UBS se ampliam e contemplam especialidades necessárias para assistência e cuidados de doenças, dentre elas, as crônicas não transmissíveis ${ }^{2}$

É conhecido que, com o passar dos anos, vem ocorrendo o aumento do número de doenças crônicas não transmissíveis na população, sendo que os dados da Organização Mundial de Saúde (OMS) mostram cerca de 36 milhões de mortes anuais decorrentes destas doenças ${ }^{3,4}$. Para os sobreviventes, as incapacidades e limitações na funcionalidade podem ser extensas assim como a qualidade de vida que sofre modificações com consequências negativas que, como em um ciclo, alteram sua saúde inclusive com interferências no aspecto emocional e social ${ }^{5,6}$.

Neste cenário, a atuação do fisioterapeuta vem ganhando espaço na área da saúde pública por ser capaz de desenvolver tratamentos curativos e intervenções preventivas, trabalhando tanto na promoção e prevenção de saúde como na reabilitação física e funcional ${ }^{7}$. A demanda para o serviço de Fisioterapia em uma UBS ainda tem sido espontânea, e a partir da implantação deste serviço, no segundo semestre de 2014, pelo Programa de Residência em Fisioterapia da Faculdade de Fisioterapia da Universidade Estadual Paulista (UNESP), houve uma maior procura por usuários da UBS, do município de Presidente Prudente/SP. Neste sentido, verificar o perfil desta demanda é importante para que a organização, estrutura e referências dentro deste serviço possam ser revisadas com o intuito de melhorar o processo de atendimento e assistência à população. Para a Fisioterapia, torna-se relevante subsidiar suas ações, inclusive em educação em saúde assim como aprimorar o conhecimento das doenças mais evidentes na população e direcionar técnicas e trabalhos a necessidade do serviço. Diante dos fatos expostos, o presente estudo teve como objetivo avaliar o perfil de usuários atendidos no serviço de Fisioterapia da UBS, em Presidente Prudente/SP para conhecer a demanda local e as necessidades do serviço.

\section{METODOLOGIA}

Trata-se de um estudo retrospectivo, realizado por análise de prontuários dos pacientes que procuraram o serviço de Fisioterapia de maneira espontânea ou após encaminhamento médico. Foram coletados dados de gênero, idade, profissão dos usuários, além do motivo do atendimento (patologia) no serviço de Fisioterapia da UBS, no município de Presidente Prudente-SP, no período de agosto de 2014 a maio de 2016. Posteriormente, foi criado um banco de dados onde foram tabulados e analisados utilizando o software Excel, apresentando dados absolutos e em percentuais das variáveis analisadas. Este estudo foi aprovado pelo Comitê de Ética e Pesquisa da FCT/UNESP, (CAAE: 05466712.4.0000.5402).

\section{RESULTADOS}

Durante o período que corresponde ao segundo semestre de 2014 ao primeiro semestre de 2016, verificou-se que 212 pessoas foram atendidas no setor de Fisioterapia da UBS, sendo $67,66 \%$ mulheres e $32,34 \%$ homens. A média de idade foi de $48,8 \pm 18,1$ anos. Com relação às profissões, as que mais apareceram foram Trabalhadores do Lar, Faxineiros, Motoristas, Operadores de máquinas e Pedreiros/Auxiliares.

A área de assistência que mais teve atendimento foi Ortopedia e Traumatologia sendo bem maior do que as áreas de ginecologia, neurologia e pneumologia. Houve, inclusive, 114 casos crônicos e 78 de lesões agudas. Dentro destas áreas, houve diferentes diagnósticos, sendo estas patologias (ou segmentos mais acometidos) apresentadas na Tabela 1.

Além das áreas citadas acima, houve três pessoas que tiveram atendimento em grupo em atividade física como promoção e manutenção em saúde.

\section{DISCUSSÃO}

O perfil dos usuários da UBS-COHAB, observado neste trabalho, foi de ambos os sexos, sendo sua maioria composto por indivíduos do gênero feminino. A maioria era trabalhador na ativa, do lar ou como 
empregado, com idade em torno de 50 anos e com necessidades de Fisioterapia na área de Ortopedia. 0 perfil dos indivíduos apresentado neste estudo condiz com a realidade da população brasileira que, em processo de envelhecimento associado a sobrecarga de trabalho, favorece o aparecimento de doenças crônicas não transmissíveis que, segundo Machado (2004), cresce nesta faixa etária e outras morbidades, como hipertensão ou diabetes, predominam em épocas mais tardias. Neste trabalho, houve mais intensidade de doenças da área de traumato-ortopedia possivelmente pela idade e relação com trabalho ${ }^{8}$. As profissões mais frequentes da população analisada neste estudo refletem a sobrecarga de trabalho, movimentos repetitivos e manutenção de uma mesma postura, muitas vezes, viciosa e por tempos prolongados. Podemos citar, entre estes, trabalhador doméstico/faxineiro, motorista e operador de máquinas. Nesta direção, compareceram problemas ligados a coluna, tornozelo e pé ${ }^{\text {. }}$

Tabela 1. Relação do número de acometimentos por área de atendimento fisioterapêutico ( $n=223)$.

\begin{tabular}{llc}
\hline Área & Acometimento & Contagem \\
\hline Ortopedia e Traumatologia & Membros Superiores $^{1}$ & 65 \\
& Coluna $^{2}$ & 45 \\
& Membros Inferiores $^{3}$ & 69 \\
& Sistêmicas $^{4}$ & 13 \\
\hline Total & & 192 \\
\hline Neurologia & AVC & 4 \\
& Outras $^{5}$ & 4 \\
\hline Total & & 8 \\
\hline Pneumologia & DPOC & 1 \\
\hline Total & & 1 \\
\hline Ginecologia e Obstetrícia / & Gestantes & 17 \\
Saúde da Mulher & Pós-operatório de Mastectomia & 1 \\
\hline Total & Incontinência Urinária & 4 \\
\hline
\end{tabular}

1: Tendinopatia de ombro, fratura de úmero, rádio ou ulna, bursite de ombro, dentre outros; 2: Hérnia discal, artrose da coluna, lombociatalgia, cervicalgia, dentre outros; 3: Fratura de fêmur, tíbia ou calcâneo, esporão de calcâneo, gonartrose, luxação de patela, dentre outros; 5: Paraparesia crural, Paralisia facial, Parkinson;

Outra situação que pode prevalecer é que a Fisioterapia ainda é bem mais conhecida na área da ortopedia e tanto pacientes como profissionais desconhecem as diferentes ações que podem ser executadas pelo Fisioterapeuta como na área de cardiologia, de pneumologia e na saúde coletiva como um todo $^{10}$

Se pensarmos na maior participação de mulheres, podemos considerar que a frequência pode ser pelo fato da mulher recorrer mais aos serviços de saúde pela maior percepção de seu estado de saúde ${ }^{11}$ ou ainda pela disponibilidade de tempo por muitas serem trabalhadoras do lar e terem o período da tarde livre. Outro estudo, realizado por Gomes, demonstra pouca procura por serviços de atenção básica por parte dos homens, sendo a questão cultural apontada como dificultadora para a prevenção ${ }^{12}$.

Historicamente, indivíduos do gênero feminino cuidam mais da saúde e procuram assistência médica quando necessário, ao contrário da maioria dos indivíduos do gênero oposto ${ }^{13}$. Ainda, segundo Barros $^{14}$, o predomínio de mulheres a partir dos 40 anos deve-se ao fato de serem mais acometidas por patologias crônicas, além de tenderem a sobreviver mais que os homens, que são mais afetados pela violência e por doenças de caráter agudo. No entanto, este estudo não buscou estes pontos, que poderiam ser explorados melhor em outros estudos, pois homens e mulheres podem ter a necessidades de fisioterapia, porém em quadros clínicos diferentes. 
Uma questão que podemos levantar como resultado da reflexão deste estudo é que na UBS o trabalho desenvolvido pelo Fisioterapeuta pode ainda estar marcado pela sua história, em que prevalece o atendimento na área de uma especialidade, e não intervenções mais complexas que envolvam prevenção de agravos ou educação em saúde. Neste conjunto, embora o serviço de Fisioterapia tenha sido implantado na unidade de estudo e integre ações educacionais, palestras, campanhas e orientações aos usuários, a característica de atendimento ainda mescla ações curativas e preventivas, o que foi demonstrado pelo perfil de usuário aqui investigado. Estas ações estão em processo de desenvolvimento e poderão ter outra abrangência e complexidade, sendo a expansão destas influenciada pela formação do Fisioterapeuta e pela contribuição do Programa de Residência. Por outro lado, o Fisioterapeuta ainda não integra o quadro de profissionais como obrigatoriedade de contratação pelos municípios nos locais de atendimento ou atenção básica. Em um estudo de 2009, Almeida já apresentava que havia pouca atuação do Fisioterapeuta na atenção básica, apontando que apenas $15 \%$ dos profissionais atuavam exclusivamente no setor público de saúde ${ }^{15}$. Dados anteriores mostravam que o papel do Fisioterapeuta na equipe de saúde pública já vinha remediada pela sua história ligada à reabilitação, entretanto observa-se modificações neste contexto, principalmente pela participação deste profissional nas equipes básicas de saúde ${ }^{16,17}$. Claro que, nestas unidades, ainda mescla-se a atuação curativa com ações de prevenção e promoção da saúde, articuladas com as informações e educação em saúde, o que condiz com as propostas do SUS.

A atenção básica envolve atendimentos que, muitas vezes, utilizam baixo custo tecnológico, embora podendo atingir outras complexidades que envolvam a manutenção da saúde como conhecimento e práticas de diferentes áreas. Esta é uma referência de proximidade com a comunidade e, embora a UBS tenha, conceitualmente, grandes diferenças dos Programas (PSF) ou Estratégias de Saúde da Família (ESF), as UBS fazem parte de uma rede de atenção em saúde, sendo uma porta de entrada, de referência e contrarreferência, e ainda desenvolvendo ações dentro da própria unidade, como o serviço de Fisioterapia configurado na UBS.

Outros trabalhos poderão ainda ser realizados para verificar as possibilidades do serviço de Fisioterapia como intervenção e seus resultados clínicos, funcionais ou na qualidade de vida da população. Também explorar o perfil do trabalho destas pessoas e de suas consequências nos agravos em saúde seria de grande valia para adequar o papel da Fisioterapia como prevenção, orientação e educação em saúde destes trabalhadores ${ }^{18}$.

\section{CONCLUSÃO}

Sendo assim, pode-se concluir que o serviço de fisioterapia realizado na UBS-COHAB teve prevalência de indivíduos do gênero feminino, trabalhadores do lar, apresentando disfunções ortopédicas, com sinais crônicos e acometimento nos segmentos de Membros Inferiores, seguidos de Membros Superiores e Coluna.

\section{CONFLITO DE INTERESSE}

Os autores declaram não haver qualquer potencial de conflito de interesse que possa interferir na imparcialidade deste trabalho científico.

\section{REFERÊNCIAS}

1. Fernandes JD, Silva RMO, Teixeira GA, Florencio RMS, Silva LS, Rebouças LCC. Aderência de cursos de graduação em enfermagem às diretrizes curriculares nacionais na perspectiva do sistema único de saúde. Esc Anna Nery. 2013; 17(1). DOI: http://dx.doi.org/10.1590/S141481452013000100012

2. Trindade $\mathrm{KMC}$, Schmitt $\mathrm{ACB}$, Casarotto RA. Queixas musculoesqueléticas em uma Unidade Básica de Saúde: implicações para o planejamento das ações em saúde e fisioterapia. Fisioter pesqui. 2013;20(3):228-34. DOI: http://dx.doi.org/10.1590/S1809-29502013000300006 
3. Santos FAZ, Netto JSL, Ramos JCL, Soares FO.Perfil epidemiológico dos atendidos pela fisioterapia no Programa Saúde e Reabilitação na Família em Camaragibe, PE. Fisioter. pesqui. 2007;14(3):50-4. DOI: http://dx.doi.org/10.1590/fpusp.v14i3.76111

4. Malta DC, Júnior JBS. O Plano de Ações Estratégicas para o Enfrentamento das Doenças Crônicas Não Transmissíveis no Brasil e a definição das metas globais para o enfrentamento dessas doenças atés2025: uma revisão. Epidemiol Ser. Saúde. 2013;22(1). DOI: http://dx.doi.org/10.5123/S1679-49742013000100016

5. Campos MO, Neto JFR. Qualidade de vida: um instrumento para promoção de saúde. Rev Baiana Saúde Públ. 2008;32(2):232-40.

6. Segre M, Ferraz FC. O conceito de Saúde. Rev Saúde Públ. 1997;31(5):538-42.DOI: http://dx.doi.org/10.1590/S0034-89101997000600016

7. Menegazzo IR, Pereira MR, Villalba JP. Levantamento epidemiológico de doenças relacionadas àsfisioterapia em uma Unidade Básica de Saúde do município de Campinas. J Health Sci Inst. 2010;28(4):348-51.

8. Machado GPM, Barreto SM, Passos VMA, Lima-Costa MF. Projeto Bambuí: prevalência de sintomas articulares crônicos em idosos. Rev Assoc Med Bras. 2004;50(4):367-72.

9. Silva, GGD., Sirena, SA. Profile of patients referred to physiotherapy by a Primary Health Care Service. Epidemiol Serv Saúde. 2015;24(1):123-33.

10. Travassos C, Viacava F, Pinheiro R, Brito A. Utilização dos serviços de saúde no Brasil: gênero, características familiares e condição social. Rev Panam Salud Publica. 2002;11(5-6):365-73. DOI:

11. Lima-Costa MF, Firmo JOA, Uchôa E. A estrutura da auto-avaliação da saúde entre os idosos: projeto Bambui. Rev Saude Publica. 2004;38(6):202-8.

http://dx.doi.org/10.1590/S0034-89102004000600011

12. Gomes R, Nascimento EF, Araújo FC. Por que os homens buscam menos os serviços de saúde que as mulheres? As explicações de homens com baixa escolaridade e com ensino superior. Cad Saude Publ. 2007;23(3):565-74.

13. Sala A, Cohen DD, Dalmaso ASW, Lima AMM, Teixeira RR. Avaliação do processo de atendimento a pacientes portadores de doença crônico- degenerativa em uma unidade basica de saude. Rev Saúde Públ. 1993;27(6):463-71.

14. Barros MBA, César GLC, Carandini L, Torre GD. Desigualdades sociais na prevalência de doenças crônicas no Brasil, PNAD-2003. Ciênc Saúde Col. 2006;11(4):911-26.

15. Almeida AL, Guimarães RB. O lugar social do fisioterapeuta. Fisioter. pesqui. 2009; 16(1):82-8.

16. Neuwald MF, Alvarenga LF. Fisioterapia e educação em saúde: investigando um serviço ambulatorial so SUS. Boletim da Saúde. Porto Alegre. 2015;19(2). 
17. Silva DJ, Da Ros MA. Inserção de profissionais de fisioterapia na equipe de saúde da família e Sistema Único de Saúde: desafios na formação. Ciênc Saúde Coletiva. 2007;12(6):1673-81.

18. Sistema de Monitoramento e Avaliação do SUS a partir da Atenção Básica no Estado de São Paulo. Temas em Saúde Coletiva, 4. São Paulo: Instituto de Saúde, 2006. p.84. Disponível em: http://www.saude.sp.gov.br/resources/instituto-de-saude/homepage/temas-saudecoletiva/pdfs/sismasus_4.pdf. 